\title{
Digestión anaerobia de efluentes de fosas sépticas
}

\section{Anaerobic Digestion of Effluents from Septic Tanks}

\author{
Méndez-Novelo R.I. \\ Ingeniería Ambiental de la Facultad de Ingeniería \\ Universidad Autónoma de Yucatán \\ Correo:mnovelo@uady.mx \\ Chan-Gutiérrez E.A. \\ Ingeniería Ambiental de la Facultad de Ingeniería \\ Universidad Autónoma de Yucatán \\ Correo:enrique_gutierrez@hotmail.com \\ Castillo-Borges E.R. \\ Ingeniería Ambiental de la Facultad de Ingeniería \\ Universidad Autónoma de Yucatán \\ Correo:cborges@uady.mx
}

Vázquez-Borges E.R.

Facultad de Ingeniería

Universidad Autónoma de Yucatán

Correo:vborges@uady.mx

Espadas-Solís A.E.

Facultad de Ingeniería

Universidad Autónoma de Yucatán

Correo:esolis@uady.mx

Información del artículo: recibido: septiembre de 2009, reevaluado: septiembre de 2010, aceptado: agosto de 2011

\section{Resumen}

En el presente trabajo se utilizó la digestión anaerobia por medio de un reactor UASB para el tratamiento de los efluentes de fosas sépticas de aguas residuales domésticas. Se diseñó el reactor con base en un tiempo de retención hidráulico (TRH) de $24 \mathrm{~h}$ y un volumen total de reactor de $24 \mathrm{~L}$. Como una modificación al reactor UASB, se colocó un filtro de gravilla en la parte superior, antes de la salida del efluente. Se modificó la metodología de arranque, inoculando el reactor con un exceso de biomasa, en este caso, líquido ruminal y re-circulando desde la parte media del reactor hasta la parte baja de éste a un caudal de $4 \mathrm{~L} / \mathrm{h}$. Después de 19 días de iniciar la inoculación, empezó la producción de biogás. El reactor se alimentó de manera continua durante 71 días, a partir del día 21 con un TRH de 36 h hasta el día 53 y después con un TRH de $24 \mathrm{~h}$ hasta el día 92. Las eficiencias durante los primeros días de la alimentación continua fueron negativas, esto debido a la estrategia de arranque con exceso de inóculo. El sistema permaneció oscilante hasta el día 72, cuando se estimó que se había alcanzado la estabilidad en términos de la eficiencia de remoción de DQO total y soluble. Se obtuvieron remociones de DQO total de $56 \%$ y de DQO soluble de $72 \%$, alcanzando concentraciones promedio en el efluente de $166 \mathrm{mg} / \mathrm{L}$ de DQO total y 62 $\mathrm{mg} / \mathrm{L}$ de DQO soluble.

\section{Descriptores}

- UASB

- rumen

- fosas sépticas

- arranque

- tratamiento anaerobio

- aguas residuales 


\begin{abstract}
In this work, anaerobic digestion was used to treat domestic septic tanks effluents using a UASB reactor. A hydraulic retention time (HRT) of $24 \mathrm{~h}$ and a total volume of $24 L$ were used to design the reactor. A gravel filter in the upper part of the UASB reactor, prior to the effluent discharge, was used as a design modification. Start up methodology was also modified; the reactor was inoculated with and excess of biomass, in this case ruminal liquid was used, and it was recycled from half height of the reactor to its lower part at a flow of $4 \mathrm{~L} / \mathrm{h}$. Biogas production started after 19 days of inoculation. The reactor was then fed continuously during 71 days, from day 21 to 53 at a HRT of $36 \mathrm{~h}$ and at a HRT of $24 \mathrm{~h}$ from day 54 up to the day 92. When the continuous feeding started, efficiencies were negative due to the start up strategy, with an excess of inoculums. The system stayed fluctuating up to the day 72 , when it was estimated that stability was reached in terms of total and soluble COD efficiencies. Removal of $56 \%$ of total COD and $72 \%$ of soluble COD were obtained, reaching effluent average concentrations of $166 \mathrm{mg} / \mathrm{L}$ of total COD and $62 \mathrm{mg} / \mathrm{L}$ of soluble COD.
\end{abstract}

\section{Introducción}

La mayoría de las viviendas del estado de Yucatán, México, disponen sus aguas residuales en fosas sépticas individuales o colectivas y sus efluentes se vierten al subsuelo, por lo que impactan directamente al acuífero subyacente. En la ciudad de Mérida, capital del estado de Yucatán, la mayor parte del agua residual está siendo dispuesta directamente al subsuelo a través de tanques sépticos, sumideros, letrinas y en algunas colonias existen redes de alcantarillado sanitario conectadas a pozos profundos a través de los cuales se inyecta el agua residual al manto salino, que subyace al agua dulce. El Censo Nacional del 2000 registra que en la ciudad de Mérida, Yucatán, 138,269 viviendas disponen sus aguas residuales mediante fosas sépticas, esto representa $80.2 \%$ del total de 172,383 viviendas censadas (INEGI, 2000).

Como tratamiento biológico, las fosas sépticas únicamente remueven parte de los sólidos suspendidos y una fracción pequeña, generalmente menor que $50 \%$ de los sólidos disueltos (Stewart, 2005). Sus efluentes no cumplen con la normatividad nacional (NOM-067ECOL-1994) y sí se considera que el subsuelo es altamente permeable y que el acuífero se encuentra a muy baja profundidad (de 1 a $40 \mathrm{~m}$ ), se puede concluir que representa un importante aporte de contaminación al acuífero.

Por estos motivos, en la convocatoria 2005 de Fondos Mixtos Gobierno del Estado de Yucatán-CONACYT, se incluyó como una de las demandas específicas determinar una alternativa eficiente de tratamiento a los efluentes de fosas sépticas. Uno de los tratamientos ensayados debía ser anaerobio, por lo que se realizó el estudio con los resultados que se presentan. Si bien, los procesos anaerobios, tienen como una de sus características que no pueden eliminar totalmente la materia orgánica y por lo tanto en muchos casos requieren un tratamiento posterior. Existen en la literatura científica experiencias exitosas de tratamientos anaerobios de aguas residuales domésticas a tiempos de retención hidráulica bajos -4 a 6 horas- (Hermes y Campos, 2003; Ghangrekar et al., 2005; Foresti et al., 2006; Collins et al., 1998). Otra característica de los procesos anaerobios es el largo periodo requerido para su arranque. Para el caso de aguas residuales domésticas, si no se usan inóculos, puede tardar hasta 8 meses (Ali et al., 2007; Florencio et al., 2001).

El grupo de investigación de la Facultad de Ingeniería de la Universidad Autónoma de Yucatán, ha ensayado con éxito el arranque de reactores anaerobios para diferentes sustratos, utilizando líquido ruminal como inóculo, por lo que se ensayó esta biomasa en el presente trabajo (Vázquez et al., 1997; Méndez et al., 2004). El rumen se puede considerar como un fermentador de temperatura constante que presenta condiciones anaerobias. Cada mililitro de contenido ruminal alberga alrededor de 10,000 a 50,000 millones de bacterias. Las bacterias se encuentran en una gran variedad de géneros y especies, por lo menos 28 especies funcionalmente importantes, las cuales se agrupan de acuerdo a su actividad. La mayoría de éstas son anaerobias estrictas, que no pueden sobrevivir en presencia de oxígeno; sin embargo, también se encuentran presentes organismos facultativos.

Aunque los sistemas anaerobios de tratamiento de aguas residuales se conocen desde el siglo pasado, se consideraron ineficientes y lentos para la necesidad del 
tratamiento de los crecientes volúmenes de aguas residuales, especialmente en áreas industriales densamente pobladas. Sin embargo, recientes desarrollos han demostrado que los procesos anaerobios son una alternativa económicamente atractiva para el tratamiento de diferentes tipos de aguas residuales industriales y aguas residuales domésticas en zonas semi-tropicales y tropicales (Van-Haandel y Lettinga, 1994).

Los sistemas modernos de tratamiento anaerobio, también llamados sistemas de segunda generación, tienen un mecanismo de retención de lodo, que los distingue de los sistemas clásicos. La retención de lodo es tan importante que los sistemas modernos son generalmente clasificados según el mecanismo que permite la retención. Existen básicamente dos mecanismos para retención de lodos en los sistemas de tratamiento de alta tasa (Van Haandel y Lettinga, 1994):

Inmovilización de lodo a través de adherencia a un material inerte de soporte

En esta categoría están: el filtro anaerobio de flujo ascendente o el descendente, y el reactor de lecho fluidizado o expandido.

\section{Separación sólido-líquido del afluente con retorno de los sólidos separados al reactor}

En esta categoría están los procesos de contacto con un decantador externo o el reactor Upflow anaerobic sludge blanket (UASB) con un decantador interno. Casos especiales son:

1) cuando el reactor anaerobio también es decantador, es decir, cuando no hay un dispositivo especial de separación de las zonas de digestión y decantación $\mathrm{y}$

2) cuando los flocs de los lodos también funcionan como los gránulos de un lecho expandido o llamado lecho de lodo granulado expandido.

El reactor UASB (figura 1) es un dispositivo que consiste en un recipiente de flujo ascendente caracterizado por el separador gas, sólido, líquido (GSL). Este separador se coloca en el reactor y divide la parte inferior o zona de digestión, donde hay un lecho (manto) de lodos responsable de la digestión anaerobia y una parte superior o zona de sedimentación. El agua residual ingresa por el fondo del reactor y sigue una trayectoria ascendente, pasando por la zona de digestión, atravesando una zona de fluidificación, en la que parte de la materia sólida es suspendida por el flujo de agua o de gases, posteriormente el agua residual entra a la zona de sedimentación. La materia orgánica del agua residual se mezcla con el lodo anaerobio en la zona de digestión, permitiendo la digestión anaerobia que resulta en la producción de gas y el aumento del volumen de lodo.

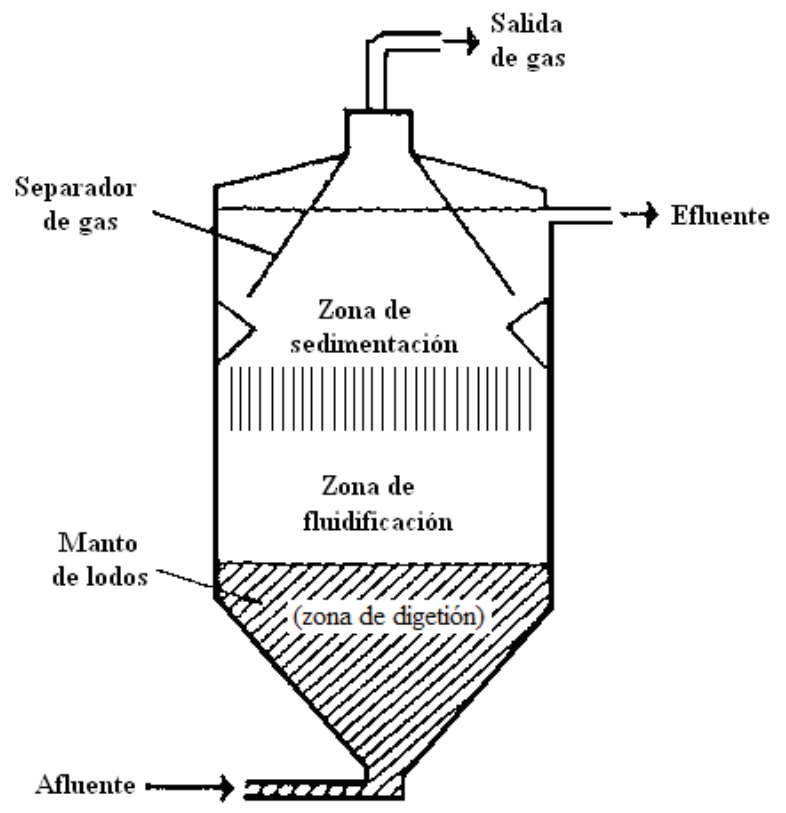

Figura 1. Esquema de un reactor UASB

En el decantador se procura retener los flocs de lodo evitando de esta manera la remoción de la biomasa activa. Cuando se acumula una cantidad suficientemente grande de sólidos en el decantador, su peso aparente se tornará mayor que la fuerza de adherencia, de modo que éstos se deslizarán entrando nuevamente a la zona de digestión en la parte inferior del reactor. De esta manera, la presencia de una zona de sedimentación en la parte superior del UASB brinda un doble beneficio, retiene los lodos, permitiendo la presencia de una gran masa en la zona de digestión, lo que a su vez permite un efluente libre de sólidos sedimentables.

Las burbujas de biogás que se forman en la zona de digestión, suben a la fase líquida donde encuentran una interfase líquido-gas, presente debajo del separador GSL. En esta interfase las burbujas se desprenden, formando una fase gaseosa. Los flocs de lodos eventualmente adheridos a las burbujas pueden ascender hasta la interfase, pero al desprenderse del gas caen para ser parte nuevamente del manto de lodos en la zona de digestión.

En general, los procesos anaerobios producen bajas concentraciones de biomasa (SSV) comparadas con los 


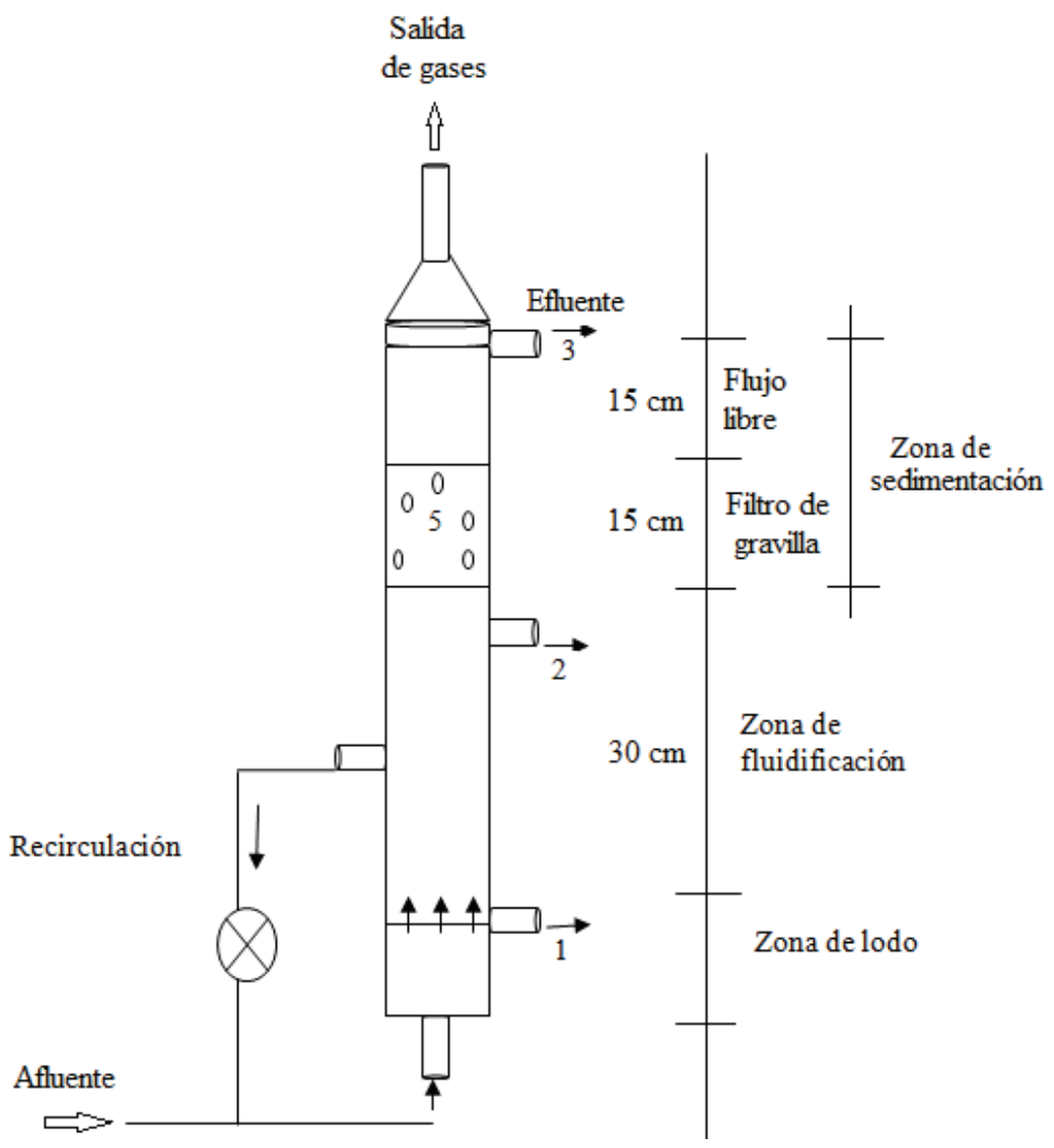

Figura 2. Esquema del reactor UASB

$1.20 \mathrm{~m}$ de altura). Se consideraron $30 \mathrm{~cm}$ para la zona de manto de lodos que es la zona en la que se realiza la recirculación, $30 \mathrm{~cm}$ para la zona de fluidificación y $30 \mathrm{~cm}$ para la zona de separación dividida en dos secciones de $15 \mathrm{~cm}$ cada una, la primera de un filtro de gravilla $\left(0.25^{\prime \prime}\right)$ y la segunda de flujo libre. En la parte superior se ensayó un cono invertido para colectar el biogás y conducirlo a una trampa de gas, para medir el volumen producido. Se instalaron válvulas a cada $30 \mathrm{~cm}$ para medir la dispersión de sólidos en el reactor. Los reactores anaerobios se arrancan haciéndolos funcionar con flujo continuo a valores altos de TRH y conforme se incrementa la eficiencia de remoción de materia orgánica se incrementa el gasto (Borzaconi, 2000; KuanYeou et al., 2004; Ali et al., 2007; Zheng y Ho, 2002).

Tratándose de aguas diluidas, la producción de biomasa activa es muy lenta, por lo que el periodo de arranque se incrementa. Por este motivo, se probó una estrategia de arranque diferente para reducir el tiempo de estabilización. Se inoculó

procesos aerobios, pero tratándose de aguas diluidas, la formación de biomasa se hace muy lenta. Por estos motivos, se ensayaron dos modificaciones: una hidráulica en el diseño del reactor y otra sobre la estrategia de arranque. La primera consiste en propiciar, mediante una recirculación y un filtro de gravilla, que la biomasa quede retenida en la parte baja del reactor y la segunda, utilizar líquido ruminal como inóculo para que una vez aclimatado el sustrato, se inicie la alimentación del agua a tratar con el TRH deseado.

Los objetivos de este estudio fueron determinar la eficiencia de remoción de materia orgánica de un proceso de digestión anaerobia de efluentes de fosa séptica, así como determinar el tiempo de arranque de un reactor UASB para el tratamiento de aguas residuales diluidas, usando líquido ruminal como inóculo.

\section{Metodología}

Se construyó un reactor UASB (figura 2) de forma cilíndrica de $15.2 \mathrm{~L}$ de capacidad (12.7 cm de diámetro y el reactor con 8 litros de líquido ruminal y se completó con el agua residual. Posteriormente, se re-circuló la biomasa a una velocidad de flujo ascendente de $1 \mathrm{~m} / \mathrm{h}$, por lo que el flujo de la bomba se ajustó a un gasto de $2.12 \mathrm{~L} / \mathrm{min}$. Se mantuvo la recirculación hasta que se comenzó a generar biogás. Luego de tres días de producir biogás, se inició la alimentación del sustrato con un flujo tal que el TRH fue de 36 horas, mismo que se continuó hasta el día 53 en el que el flujo se incrementó hasta alcanzar un TRH de 24 horas, con el que se logró alcanzar un estado estable (figura 3).

Para el monitoreo del reactor se midieron a la entrada y salida cada dos o tres días, el $\mathrm{pH}$, la temperatura, los SST, los SSV, la alcalinidad, la DQO total, la DQO soluble y la $\mathrm{DBO}_{5}$, de acuerdo con el Standard Methods (2005).

Cuando se concluyó la fase experimental del reactor UASB, se midieron la velocidad de asentamiento de la interfase de lodos y la distribución de sólidos (ST, SST, SV y SSV), pH, alcalinidad y DQO total y soluble a lo largo del reactor. Para tal efecto, se removieron los lo- 


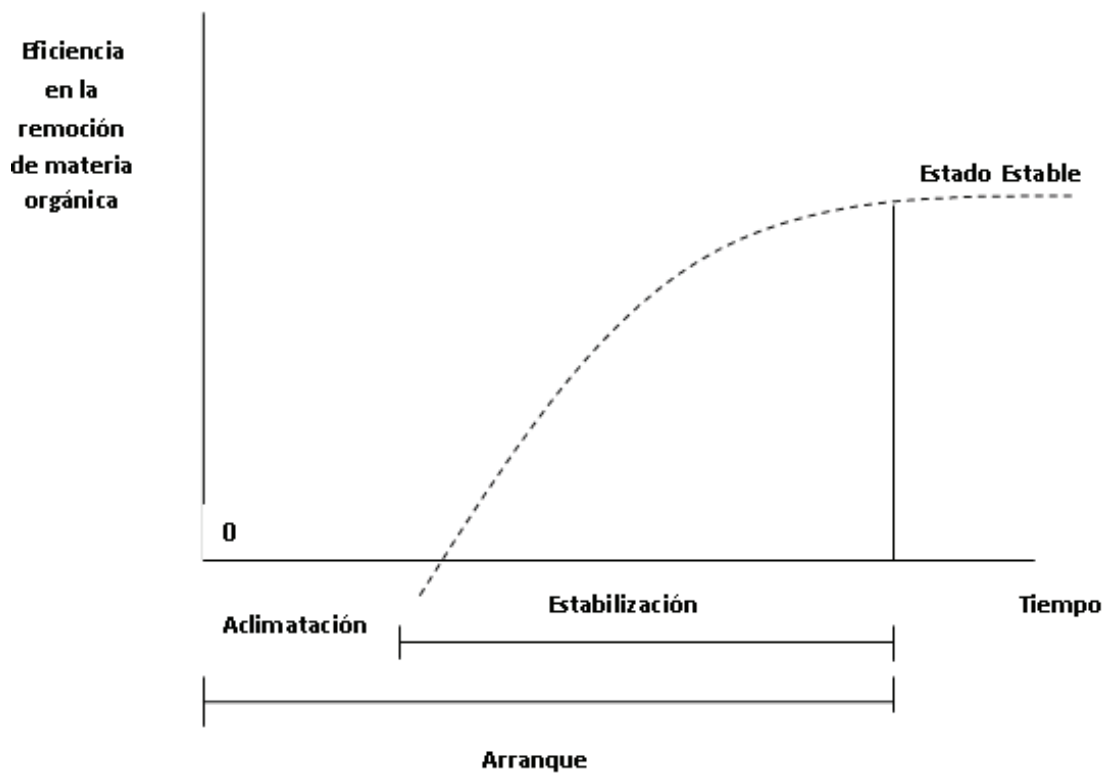

Figura 3. Estrategia de arranque del reactor UASB

dos del fondo del reactor y se vertió 1 litro de éste en una probeta graduada de $1000 \mathrm{ml}$, la cual cuenta con una altura de $34 \mathrm{~cm}$. Posteriormente, se dejó sedimentar y se midió el volumen que ocupaba el lodo a diferentes tiempos, con lo que se determinó la velocidad de asentamiento del manto de lodos, dividiendo la variación de la altura de la interfase entre la variación del tiempo en que ésta se realizó.

\section{Resultados y discusión}

Al iniciar el proceso anaerobio, se caracterizó la mezcla rumen-agua residual, obteniéndose los valores que se presentan en la tabla1.

A los 19 días de iniciada la recirculación, comenzó la producción de biogás, con lo que se consideró concluida la etapa de aclimatación (figura 3). Del día 21 al 53, se trabajó con el TRH de 36 horas y del día 55 al 92 con el TRH de 24 horas. En las tablas 2 y 3 se muestran los resultados de los análisis de DQO total y soluble, así como de los sólidos (ST, STV, SST y SSV).

Durante los primeros 11 días de alimentación continua del sustrato (del 21 al 32), los valores de la DQO (total y soluble) del efluente fueron superiores a los del

Tabla 1. Caracterización de la mezcla rumen-agua residual

\begin{tabular}{lcccccccc}
\hline Parámetro & $\mathrm{ST}$ & $\mathrm{STV}$ & $\mathrm{SF}$ & $\mathrm{SST}$ & $\mathrm{SSV}$ & $\mathrm{DQOt}$ & $\mathrm{pH}$ & Temperatura \\
\hline Unidad & $\mathrm{mg} / \mathrm{L}$ & $\mathrm{mg} / \mathrm{L}$ & $\mathrm{mg} / \mathrm{L}$ & $\mathrm{mg} / \mathrm{L}$ & $\mathrm{mg} / \mathrm{L}$ & $\mathrm{mg} / \mathrm{L}$ & --- & ${ }^{\circ} \mathrm{C}$ \\
Valor & 8916 & 3796 & 5120 & 1220 & 980 & 10000 & 6.96 & 24.4 \\
\hline
\end{tabular}

afluente, dado que se desplazaba parte de la biomasa del reactor, pero a partir del día 35 los valores de materia orgánica del efluente, medidos como DQO (total y soluble), comienzan a ser inferiores a los del afluente.

No obstante, la eficiencia de remoción es muy variable hasta el día 74, cuando se estabiliza el sistema y se obtiene a partir de ese tiempo una eficiencia de remoción promedio de $56 \%$ de $\mathrm{DQO}_{\mathrm{t}}$ y de $72 \%$ de $\mathrm{DQO}_{\mathrm{s}}$. Las concentraciones promedio en el efluente fueron de $166 \mathrm{mg} / \mathrm{L}$ para a $\mathrm{DQO}_{\mathrm{t}}$ y $62 \mathrm{mg} / \mathrm{L}$ para la $\mathrm{DQO}_{\mathrm{s}}$.

De manera semejante, durante los primeros 7 días de alimentación continua del sustrato (de los días 21 al 28) los valores de los SSV del efluente fueron superiores a los del afluente, por lo que no se obtuvieron remociones, pero a partir del día 30, se obtuvieron remociones de SSV y de SST.

El valor promedio de las eficiencias de remoción de SSV y SST del día 74 al 92 fue $62 \%$ y $61 \%$, respectivamente, obteniendo concentraciones en el efluente de 17 $\mathrm{mg} / \mathrm{L}$ de SSV y de $31 \mathrm{mg} / \mathrm{L}$ de SST. La alta retención de SSV evidencia que el sistema hidráulico (filtro de grava) es adecuado para retener la biomasa activa. Los valores de remoción de SST obtenidos son semejantes al valor promedio de los obtenidos de varios estudios de tratamientos anaerobios a aguas residuales domésticas presentados en la tabla 4.

De acuerdo con Morgan et al. (1996), un reactor UASB alimentado con agua residual municipal típica con temperatura superior a los $20^{\circ} \mathrm{C}$, entregará una calidad de agua tratada con DQO total entre 140 y $160 \mathrm{mg} / \mathrm{L}$ y SST entre 50 y $60 \mathrm{mg} / \mathrm{L}$. En nuestro caso, el agua residual municipal no era "típica", dado que había estado sometida a un proceso de depuración (fosa séptica), por lo que se eliminaba parte de su carga orgánica, suspendida y disuelta. Por estos motivos, no se podían esperar altas remociones de materia orgánica en el proceso anaerobio, pero se debía determinar si los efluentes podían cumplir con las concentraciones de descarga establecidas en la normatividad mexicana. No obstante, las concentraciones promedio en el efluente (166 $\mathrm{mg} / \mathrm{L}$ de $\mathrm{DQO}_{\mathrm{t}}$ y $95 \mathrm{mg} / \mathrm{L}$ de SST) son semejantes a las propuestas por Morgan et al. (1996). 
DOI: http://dx.doi.org/10.22201/fi.25940732e.2012.13n3.032

Digestión anaerobia de efluentes de fosas sépticas

\begin{tabular}{|c|c|c|c|c|c|c|c|c|}
\hline \multirow{2}{*}{$\begin{array}{l}\text { Tiempo } \\
\text { (días) }\end{array}$} & \multicolumn{2}{|c|}{$\mathrm{pH}$} & \multicolumn{3}{|c|}{$\mathrm{DQO}_{\mathrm{t}}(\mathrm{mg} / \mathrm{L})$} & \multicolumn{3}{|c|}{$\mathrm{DQO}_{\mathrm{s}}(\mathrm{mg} / \mathrm{L})$} \\
\hline & A & E & A & E & $\% \mathrm{R}$ & A & E & $\% \mathrm{R}$ \\
\hline 21 & 7.61 & 7.45 & 138 & 2320 & --- & 49 & 426 & --- \\
\hline 23 & 7.74 & 7.36 & 142 & 1740 & --- & 53 & 382 & --- \\
\hline 25 & 7.31 & 7.43 & 129 & 1320 & --- & 65 & 320 & --- \\
\hline 28 & 7.45 & 7.54 & 126 & 938 & --- & 51 & 123 & --- \\
\hline 30 & 7.85 & 7.67 & 115 & 340 & --- & 60 & 108 & --- \\
\hline 32 & 7.68 & 7.7 & 157 & 345 & --- & 68 & 70 & --- \\
\hline 35 & 7.42 & 7.53 & 160 & 97 & 40 & 113 & 40 & 65 \\
\hline 37 & 8.01 & 8.05 & 120 & 113 & 6 & 70 & 43 & 39 \\
\hline 39 & 7.45 & 7.61 & 236 & 110 & 53 & 93 & 35 & 62 \\
\hline 42 & 7.3 & 7.57 & 156 & 67 & 57 & 57 & 21 & 63 \\
\hline 44 & 7.5 & 7.44 & 126 & 84 & 33 & 90 & 44 & 51 \\
\hline 46 & 7.46 & 7.8 & 213 & 103 & 52 & 96 & 36 & 63 \\
\hline 49 & 7.36 & 7.5 & 122 & 79 & 35 & 170 & 90 & 47 \\
\hline 51 & 7.38 & 7.85 & 170 & 142 & 16 & 123 & 77 & 37 \\
\hline 53 & 7.49 & 7.65 & 206 & 131 & 36 & 171 & 98 & 43 \\
\hline 56 & 7.66 & 7.82 & 195 & 147 & 25 & 127 & 89 & 30 \\
\hline 58 & 8.01 & 8.12 & 345 & 140 & 59 & 208 & 78 & 63 \\
\hline 60 & 7.81 & 7.92 & 205 & 110 & 46 & 177 & 74 & 58 \\
\hline 63 & 8.12 & 8.2 & 155 & 112 & 28 & 120 & 68 & 43 \\
\hline 65 & 8.15 & 8.02 & 172 & 121 & 30 & 95 & 47 & 51 \\
\hline 67 & 7.72 & 7.83 & 484 & 175 & 64 & 182 & 43 & 76 \\
\hline 70 & 7.45 & 7.65 & 328 & 112 & 66 & 158 & 41 & 74 \\
\hline 72 & 8.12 & 8.23 & 459 & 245 & 47 & 175 & 50 & 71 \\
\hline 74 & 7.72 & 7.78 & 457 & 201 & 56 & 161 & 35 & 78 \\
\hline 77 & 7.66 & 7.9 & 464 & 178 & 62 & 171 & 43 & 75 \\
\hline 79 & 7.84 & 7.92 & 228 & 94 & 59 & 161 & 32 & 80 \\
\hline 81 & 8.09 & 7.98 & 385 & 164 & 57 & 175 & 43 & 75 \\
\hline 84 & 7.56 & 7.77 & 300 & 152 & 49 & 176 & 52 & 70 \\
\hline 86 & 7.88 & 7.9 & 440 & 184 & 58 & 176 & 62 & 65 \\
\hline 88 & 8.13 & 8.1 & 370 & 178 & 52 & 190 & 68 & 64 \\
\hline 92 & 7.99 & 8.12 & 420 & 175 & 58 & 215 & 73 & 66 \\
\hline
\end{tabular}

Tabla 2. Resultados de $\mathrm{DQO}_{\mathrm{t}}$ y $\mathrm{DQO}_{\mathrm{s}}$ del arranque del reactor UASB

A: afluente

E: efluente 


\begin{tabular}{|c|c|c|c|c|c|c|c|c|c|c|c|c|}
\hline \multirow{2}{*}{$\begin{array}{c}\text { Tiempo } \\
\text { (días) }\end{array}$} & \multicolumn{3}{|c|}{ ST } & \multicolumn{3}{|c|}{ STV } & \multicolumn{3}{|c|}{ SST } & \multicolumn{3}{|c|}{ SSV } \\
\hline & A & E & $\% \mathrm{R}$ & A & E & $\% \mathrm{R}$ & A & E & $\% \mathrm{R}$ & A & $\mathrm{E}$ & $\% \mathrm{R}$ \\
\hline 21 & 720 & 3850 & --- & 113 & 2978 & --- & 84 & 1040 & --- & 69 & 740 & --- \\
\hline 23 & 830 & 3216 & --- & 120 & 2115 & --- & 76 & 910 & --- & 64 & 650 & --- \\
\hline 25 & 940 & 1736 & --- & 104 & 1564 & --- & 60 & 540 & --- & 48 & 440 & --- \\
\hline 28 & 916 & 1458 & --- & 76 & 560 & --- & 168 & 510 & --- & 142 & 490 & --- \\
\hline 30 & 750 & 697 & 7 & 135 & 76 & 44 & 190 & 134 & 29.5 & 112 & 82 & 27 \\
\hline 32 & 640 & 580 & 9 & 176 & 70 & 60 & 120 & 80 & 33.3 & 84 & 42 & 50 \\
\hline 35 & 595 & 556 & 7 & 148 & 58 & 61 & 127 & 56 & 55.9 & 78 & 36 & 54 \\
\hline 37 & 1245 & 1114 & 11 & 115 & 49 & 57 & 200 & 144 & 28 & 164 & 112 & 32 \\
\hline 39 & 1140 & 860 & 25 & 296 & 24 & 92 & 176 & 84 & 52 & 108 & 56 & 48 \\
\hline 42 & 804 & 732 & 9 & 156 & 40 & 74 & 52 & 32 & 38 & 28 & 16 & 43 \\
\hline 44 & 792 & 736 & 7 & 84 & 48 & 43 & 112 & 48 & 57 & 80 & 36 & 55 \\
\hline 46 & 828 & 720 & 13 & 76 & 40 & 47 & 324 & 44 & 86 & 260 & 32 & 88 \\
\hline 49 & 724 & 620 & 14 & 124 & 40 & 68 & 120 & 64 & 47 & 80 & 48 & 40 \\
\hline 51 & 796 & 488 & 39 & 152 & 48 & 68 & 132 & 60 & 55 & 81 & 50 & 38 \\
\hline 53 & 1196 & 1156 & 3 & 184 & 52 & 72 & 164 & 73 & 55 & 108 & 45 & 58 \\
\hline 56 & 1264 & 1168 & 8 & 260 & 180 & 31 & 104 & 55 & 47 & 68 & 38 & 44 \\
\hline 58 & 1116 & 1026 & 8 & 160 & 128 & 20 & 230 & 152 & 34 & 115 & 76 & 34 \\
\hline 60 & 1232 & 1138 & 8 & 172 & 128 & 26 & 160 & 59 & 63 & 56 & 27 & 52 \\
\hline 63 & 1276 & 1200 & 6 & 145 & 100 & 31 & 210 & 95 & 55 & 106 & 38 & 64 \\
\hline 65 & 1300 & 1214 & 7 & 200 & 143 & 29 & 180 & 98 & 46 & 64 & 29 & 55 \\
\hline 67 & 1268 & 1028 & 19 & 296 & 140 & 53 & 152 & 60 & 61 & 95 & 23 & 76 \\
\hline 70 & 440 & 293 & 33 & 128 & 68 & 47 & 106 & 24 & 77 & 70 & 12 & 83 \\
\hline 72 & 264 & 160 & 39 & 140 & 112 & 20 & 144 & 40 & 72 & 92 & 10 & 89 \\
\hline 74 & 332 & 228 & 31 & 176 & 124 & 30 & 230 & 16 & 93 & 170 & 4 & 98 \\
\hline 77 & 329 & 197 & 40 & 210 & 46 & 78 & 164 & 20 & 88 & 120 & 14 & 88 \\
\hline 79 & 950 & 790 & 17 & 139 & 51 & 63 & 156 & 40 & 74 & 90 & 30 & 67 \\
\hline 81 & 789 & 700 & 11 & 163 & 63 & 61 & 110 & 20 & 82 & 32 & 12 & 62 \\
\hline 84 & 1120 & 1036 & 8 & 300 & 136 & 55 & 184 & 37 & 80 & 152 & 16 & 89 \\
\hline 86 & 1115 & 1064 & 5 & 272 & 180 & 34 & 156 & 41 & 74 & 88 & 18 & 80 \\
\hline 88 & 1038 & 944 & 9 & 178 & 80 & 55 & 190 & 37 & 81 & 84 & 25 & 70 \\
\hline 92 & 785 & 685 & 13 & 121 & 78 & 36 & 127 & 35 & 72 & 79 & 18 & 77 \\
\hline
\end{tabular}

Tabla 3. Resultados de ST, STV, SST y SSV del arranque del reactor UASB

A: afluente

E: efluente 
Tabla 4. Comparación de resultados de tratamiento de aguas residuales domésticas con procesos anaerobios

\begin{tabular}{|c|c|c|c|c|c|c|c|c|c|c|}
\hline Parámetro & Unidad & 1 & \multicolumn{2}{|c|}{2} & $3^{*} 1$ & 4 & \multicolumn{2}{|c|}{5} & 6 & 7 \\
\hline $\mathrm{DQO}_{\mathrm{sa}}$ & $\mathrm{mg} / \mathrm{L}$ & 248 & --- & --- & --- & 107 & 168 & 360 & 94 & 178 \\
\hline $\mathrm{DQO}_{\mathrm{ta}}$ & $\mathrm{mg} / \mathrm{L}$ & 467 & --- & 712 & 467 & 146 & 567 & 1113 & 566 & 383 \\
\hline $\mathrm{DQO}_{\mathrm{se}}$ & $\mathrm{mg} / \mathrm{L}$ & 98 & --- & --- & --- & 15 & 84 & 110 & 53 & 51 \\
\hline $\mathrm{DQO}_{\mathrm{te}}$ & $\mathrm{mg} / \mathrm{L}$ & 155 & 191 & 144 & 174 & 49 & 190 & 295 & 119 & 166 \\
\hline Remoción $\mathrm{DQO}_{\mathrm{s}}$ & $\%$ & 57 & --- & --- & --- & 86 & 50 & 69 & 43 & 72 \\
\hline Remoción $\mathrm{DQO}_{\mathrm{t}}$ & $\%$ & 65 & 73 & 79 & 62 & 66 & 66 & 73 & 79 & 56 \\
\hline $\mathrm{TRH}$ & $\mathrm{h}$ & 8 & 9 & 7.5 & 4 a 6 & 10 & $16^{*} 2$ & $8^{*} 2$ & 14 & 24 \\
\hline Arranque & d & 200 & & & --- & 80 & 60 & 60 & 120 & 74 \\
\hline Inóculo & --- & $\begin{array}{c}\text { Incubó } 8 \\
\text { reactores } \\
\text { con lodos } \\
\text { diversos }\end{array}$ & & & & anae & & & & Rumen \\
\hline $\mathrm{SST}_{\mathrm{a}}$ & $\mathrm{mg} / \mathrm{L}$ & 180 & --- & 386 & 310 & --- & --- & --- & 560 & 165 \\
\hline $\mathrm{SST}_{\mathrm{e}}$ & $\mathrm{mg} / \mathrm{L}$ & 80 & 50 & 32 & 109 & --- & --- & --- & 402 & 31 \\
\hline
\end{tabular}

$\mathrm{DQO}_{\mathrm{sa}}$ : DQO soluble en el afluente; $\mathrm{DQO}_{\mathrm{ta}}$ : DQO total en el afluente; $\mathrm{DQO}_{\mathrm{se}}$ : DQO soluble en el efluente; DQO $\mathrm{DQ}_{\mathrm{te}}$ DQO total en el efluente; $\mathrm{SST}_{\mathrm{a}}$ : Sólidos suspendidos totales en el afluente; $\mathrm{SST}_{\mathrm{e}}$ : Sólidos suspendidos totales en el efluente

1. Florencio et al. (2001); 2. Chernicharo y Cardoso (1999); 3. Morgan et al. (1996); 4. Collins et al. (1998); 5. Elmitwalli et al. (2003); 6. Ali et al. (2007); 7. Resultados de esta investigación

*11 Valores promedio de 5 plantas de tratamiento

${ }^{*}$ Valores óptimos calculados con un modelo matemático

Los valores promedio de $\mathrm{DQO}_{\mathrm{ta}}$ y $\mathrm{DQO}_{\mathrm{sa}}$ presentados en la tabla 4 (577 mg/L y $195 \mathrm{mg} / \mathrm{L}$, respectivamente) son superiores a los obtenidos en el presente estudio (383 mg/L y $178 \mathrm{mg} / \mathrm{L}$ ), no obstante, las eficiencias de remoción de $\mathrm{DQO}_{\mathrm{s}}$ se encuentran en el rango de las reportadas en la tabla 4, mientras que los valores de remoción de $\mathrm{DQO}_{\mathrm{t}}$ obtenidos en el estudio son menores que los valores promedio de la tabla 4 . Lo anterior indica, que existe una mejor degradación de materia orgánica disuelta que de materia orgánica suspendida.

Los valores de $\mathrm{pH}$, tanto en el afluente como en el influente, se mantienen ligeramente alcalinos durante todo el proceso, lo que indica que se mantuvieron las condiciones adecuadas para el proceso anaerobio, toda vez que para que el proceso se desarrolle de forma satisfactoria, el $\mathrm{pH}$ debe estar en torno a la neutralidad, presentando problemas graves si el $\mathrm{pH}$ está por debajo de 6 , o sube por encima de 8.3 .

Florencio et al. (2001) obtuvieron un efluente de 155 $\mathrm{mg} / \mathrm{L}$ de DQO total; sin embargo, la eficiencia de remoción que consiguió en su experimento fue $56 \%$ en promedio. Chernicharo y Cardoso (1999), obtuvieron un efluente de $144 \mathrm{mg} / \mathrm{L}$ de DQO total con una DQO de entrada de $712 \mathrm{mg} / \mathrm{L}$ y una eficiencia en la remoción de $79 \%$. Existen en la literatura muchos reportes de experiencias del proceso UASB con aguas residuales domésticas en los que, en general, se reporta que se consiguen mejores eficiencias de remoción de materia orgánica cuando los valores de la DQO afluente son altos, es de- cir, dado que los valores de la DQO efluente no varían mucho (no se encontraron en la literatura consultada valores menores de $100 \mathrm{mg} / \mathrm{L}$ ), la eficiencia de remoción está directamente relacionada con la concentración del afluente.

En la tabla 5, se presentan los límites máximos permisibles de contaminantes en las descargas de aguas residuales a cuerpos receptores provenientes de los sistemas de alcantarillado o drenaje municipal en México. Los límites máximos permisibles (promedios diarios) que la NOM-067-ECOL1994 establece para la DQO, $\mathrm{DBO}_{5}$ y SST, en el caso de ciudades menores de 80,000 habitantes, son $200 \mathrm{mg} / \mathrm{L}, 100 \mathrm{mg} / \mathrm{L}$ y $100 \mathrm{mg} / \mathrm{L}$, respectivamente. Si se comparan estos valores con los de DQO, $\mathrm{DBO}_{5}$ y SST, que se reportan en la tabla 2, se concluye que el reactor UASB es capaz de alcanzar estos valores, principalmente en lo que concierne a DQO y $\mathrm{DBO}_{5}$, pero un proceso de postratamiento aseguraría definitivamente el cumplimiento de los límites de esta norma, inclusive para ciudades mayores de 80,000 habitantes. En el caso particular del presente estudio, se alcanzaron valores de sólidos y de DQO, que cumplen con lo estipulado para los límites máximos permisibles de descarga para poblaciones de menos de 80,000 habitantes, pero no para poblaciones mayores en el caso de la DQO.

El arranque de reactores anaerobios, especialmente de aguas diluidas, puede retardarse debido a que en este tipo de procesos se tiene una baja generación de 
Tabla 5. Límites máximos permisibles de descarga de aguas residuales a cuerpos receptores para centros de poblaciones

\begin{tabular}{|c|c|c|c|c|}
\hline \multirow[t]{2}{*}{ Parámetros } & \multicolumn{2}{|c|}{$\begin{array}{c}\text { Ciudades con menos de 80,000 } \\
\text { habitantes }\end{array}$} & \multicolumn{2}{|c|}{$\begin{array}{c}\text { Ciudades con más de 80,000 } \\
\text { habitantes }\end{array}$} \\
\hline & Promedio diario & Instantáneo & Promedio diario & Instantáneo \\
\hline $\mathrm{pH}$ (unidades de $\mathrm{pH}$ ) & $6-9$ & $6-9$ & $6-9$ & $6-9$ \\
\hline Sólidos suspendidos totales (mg/L) & 100 & 150 & 50 & 80 \\
\hline Grasas y aceites (mg/L) & 20 & 30 & 10 & 20 \\
\hline Sólidos sedimentables $(\mathrm{ml} / \mathrm{L})$ & 1 & 1.2 & 1 & 1.2 \\
\hline Demanda bioquímica de oxígeno $(\mathrm{mg} / \mathrm{L})$ & 100 & 150 & 50 & 80 \\
\hline Demanda química de oxígeno $(\mathrm{mg} / \mathrm{L})$ & 200 & 250 & 100 & 160 \\
\hline Substancias Activas al azul de metileno (mg/L) & 5 & 8 & 5 & 8 \\
\hline
\end{tabular}

Fuente: NOM-067-ECOL-1994

biomasa activa. Para realizar el arranque en menor tiempo deben utilizarse lodos anaerobios, que pueden ser difíciles de obtener. Por estos motivos, se ensayó la utilización de líquido ruminal como inóculo. Este líquido puede obtenerse en cualquier rastro de ganado bobino durante todo el año y ha mostrado excelentes cualidades como inóculo para procesos anaerobios de diferentes sustratos (Vázquez et al., 1997, Méndez et al., 2004).

Puede considerarse que el arranque en el reactor se concluyó a los 74 días de iniciado el proceso, lo cual resulta un tiempo corto tratándose de aguas diluidas. En la literatura consultada, en general, se requieren de tiempos mayores para alcanzar la estabilización. Collins et al. (1998) alcanzaron la estabilización a los 60 días de iniciado el proceso en aguas residuales domésticas a $20^{\circ} \mathrm{C}$ y a 80 días cuando la temperatura del agua fue de $30^{\circ} \mathrm{C}$, Elmitwalli et al. (2003) trabajando con aguas residuales domésticas obtuvieron la estabilización a los 121 días de iniciado el proceso, Florencio et al. (2001) alcanzaron la estabilización a los 200 días trabajando con aguas residuales domésticas, mientras que Ali et al. (2007), con el mismo tipo de aguas, necesitó 50 días para seleccionar el mejor inóculo, 30 días para aclimatarlo y 40 días para alcanzar el estado estable, por lo que puede concluirse que la estrategia de arranque fue adecuada.
El diseño hidráulico, consistente en ensayar un filtro de gravilla en la parte superior del reactor, resultó eficaz al propiciar que la distribución de sólidos en el reactor fuera la deseada, con concentraciones de 18636 $\mathrm{mg} / \mathrm{L}$ de ST (SSV = 10,100 mg/L) en el fondo del reactor y de $1234 \mathrm{mg} / \mathrm{L}$ de ST (SSV = $66 \mathrm{mg} / \mathrm{L}$ ) en promedio, en el resto del reactor.

El día final de la experimentación se determinaron los parámetros internos del reactor a diferentes alturas (tabla 6). Los resultados son los siguientes:

La cantidad de sólidos dentro del reactor es mucho mayor en la altura cero que en cualquier otra zona del reactor, esto es, la zona en la que se encuentra la cama de lodos, que por los resultados se puede decir que tiene un espesor menor a $30 \mathrm{~cm}$. Al igual que la cantidad de los sólidos totales, los sólidos volátiles, sólidos suspendidos totales y sólidos suspendidos volátiles son mayores a esa altura. De manera semejante, la cantidad de materia orgánica medida como $\mathrm{DQO}_{\mathrm{t}} \mathrm{y}$ la alcalinidad como $\mathrm{CaCO}_{3}$ son mayores en el fondo del reactor. Se nota un perfil de sólidos decreciente desde el fondo del reactor hacia la parte superior. Estos resultados, indican que el diseño hidráulico fue apropiado porque permitió retener la mayor parte de los ST y SSV en el fondo del reactor (zona de manto de lodos), que es donde se efectúa la mayor remoción de materia orgánica.

\begin{tabular}{cccccccccc}
\hline Altura & ST & SV & SST & SSV & DQO t & DQO s & pH & Temperatura & Alcalinidad \\
\hline 130 & 1104 & 184 & 56 & 36 & 137 & 74 & 7.42 & 25.7 & 748 \\
90 & 1308 & 220 & 100 & 68 & 223 & 165 & 7.48 & 25.6 & 768 \\
60 & 1272 & 220 & 100 & 68 & 232 & 175 & 7.47 & 25.6 & 776 \\
30 & 1332 & 224 & 132 & 92 & 235 & 168 & 7.48 & 25.5 & 812 \\
0 & 18636 & 11660 & 17820 & 10100 & 515 & 130 & 7.28 & 25.6 & 1089 \\
\hline
\end{tabular}

Tabla 6. Resultados de los parámetros internos

Altura en $\mathrm{cm}$; sólidos en $\mathrm{mg} / \mathrm{L}$; DQO en $\mathrm{mg} / \mathrm{L}$; temperatura en ${ }^{\circ} \mathrm{C}$; alcalinidad en $\mathrm{mg} \mathrm{CaCO}_{3} / \mathrm{L}$ 


\begin{tabular}{ccccccc}
\hline Tiempo (min) & 0 & 15 & 30 & 45 & 60 & 75 \\
\hline Volumen (L) & 1.000 & 0.830 & 0.730 & 0.670 & 0.610 & 0.580 \\
Altura (cm) & 34 & 28.22 & 24.82 & 22.78 & 20.74 & 19.72 \\
\hline
\end{tabular}

Tabla 7. Resultados de prueba de sedimentabilidad de lodos
Los resultados de la prueba de sedimentabilidad se presentan en la tabla 7.

La velocidad de sedimentación del lodo anaerobio fue de $0.4 \mathrm{~m} / \mathrm{h}$, lo que indica que es un lodo de poca sedimentabilidad. Souza (1986), recomienda para el diseño de reactores UASB una velocidad de sedimentación de $5 \mathrm{~m} / \mathrm{h}$. Un alta tasa de sedimentación del manto de lodos, con una velocidad de flujo ascensional baja, propicia una alta eficiencia de retención de sólidos suspendidos (al tener una menor porosidad). Debido a que la velocidad de sedimentación del lodo anaerobio alcanzada $(0.4 \mathrm{~m} / \mathrm{h})$ fue menor que la del flujo ascensional $(1 \mathrm{~m} / \mathrm{h})$, se ocasionó que las remociones de sólidos totales no fueran del todo las ideales, ya que con un lodo de poca sedimentabilidad es fácil que pueda flotar por la acción de la velocidad ascensional y por los desprendimientos de gases de la cama de lodos. Borzacconi (2000), obtuvo una velocidad de sedimentación de $6.8 \mathrm{~m} / \mathrm{h}$, superior a la recomendada por Souza para el diseño de reactores UASB. Según Borzaconni, estos valores superiores a los recomendados estarían indicando que en la zona de sedimentación de lodos del reactor no habría problemas con lavados o escape de lodos

\section{Conclusiones}

El arranque del reactor para un TRH de 24 horas se alcanzó en 74 días, obteniéndose una eficiencia de remoción de materia orgánica de $72 \%$ para $\mathrm{DQO}_{\mathrm{s}} \mathrm{y}$ de $56 \%$ para $\mathrm{DQO}_{\mathrm{t}}$.

El líquido ruminal se aclimató al sustrato (afluente de fosa séptica) en 21 días.

El diseño hidráulico del reactor permitió retener la biomasa activa en la zona de lodos del reactor.

El reactor UASB puede ser capaz de alcanzar valores de remoción de materia orgánica y sólidos, que cumplan con uno o más de los incisos de las normas aplicables en México.

\section{Referencias}

Ali M., Al-Sa'ed R., Mahmound N. Start-Up Phase Assessment of a UASB-Septic Tank System Treating Domestic Septage. The Arabian Journal for Science and Engineering, volumen 32 (número $1-C), 2007$.
APHA, AWWA, WPFC. Standard Methods for the Examination of Water and Wastewater, USA, 19a., 2005.

Borzacconi L. Seguimiento de las características de la manta de lodos en un reactor UASB piloto, en: XXVII Congresso Interamericano de Engenharia Sanitária e Ambiental, Associação Brasileira de Engenharia Sanitária e Ambiental, Porto alegre, Brasil, 2000.

Chernicharo C. y Cardoso M. Development and Evaluation of a Partitioned Upflow Anaerobic Sludge Blanket UASB Reactor o for the Treatment of Sewage from Small Villages. Water Science and Technology (número 40), 1999: 107-113.

Collins A.G., Theis T.L., Kilambi S., He L., Pavlostathis S.G. Anaerobic Treatment of Low-Strength Domestic Wastewater Using an Anaerobic Expanded Bed Reactor. Environmental Engineering, volumen 124 (número 7), 1998: 652-659.

Elmitwalli T., Al-Sarawey A., Zeeman G., El-Sherbiny M., Lettinga G. Anaerobic Biodegradability and Treatment of Egyptian Domestic Sewage. Environmental Science and Health (Part A, Toxic/Hazardous Substances \& Environmental Engineering), volumen 38 (número 10), 2003: 2043-2055.

Florencio L., Takayuki K., Cardoso de Morais J. Domestic Sewage Treatment in Full-Scale UASB Plant at Mangueira, Recife, Pernambuco. Water Science and Technology (número 44), 2001: 71-77.

Foresti E., Zaiat M. y Vallero M. Anaerobic Processes as the Core Technology for Sustainable Domestic Wastewater Treatment: Consolidated Applications, New Trends, Perspectives and Challenges. Environmental Science and Bio/Technology (número 5), 2006: 3-19.

Ghangrekar M.M., Asolekar S.R. y Joshi S.G. Characteristics of Sludge Developed Under Different Loading Conditions During UASB Reactor Start-up and Granulation. Water Research, volumen 39, 2005: 1123-1133.

Hermes P.F. y Campos J.R. Reator anaeróbio hibrido para o tratamento de esgoto sanitario, en: XXIII Congresso Brasileiro de Engenharia Sanitária e Ambiental, 2003, pp. 1-10.

INEGI. XII Censo General Nacional de Población y Vivienda 2000, México, 2000.

Méndez R., Vázquez E. y Febles H. Selección de la mejor biomasa anaerobia para el tratamiento de lixiviados. Tecnología del agua, España, (número 245), 2004: 40-47.

Morgan F., Morgan J., Noyola A. Aplicabilidad de la digestión anaerobia para el tratamiento de aguas residuales municipales, Consolidación para el desarrollo, México DF, AIDIS, 1996, pp. 1-8.

Norma Oficial Mexicana NOM-067-ECOL-1994. Límites máximos permisibles de contaminantes en las descargas de aguas resi- 
duales a cuerpos receptores provenientes de los sistemas de alcantarillado o drenaje municipal, 1994.

Rodríguez J., Sosa G.J. y Garza Y. Bioconversión anaerobia como una alternativa para la remoción de DQO contenido en aguas residuales domésticas del rastro municipal de la ciudad de Saltillo, Coahuila, México. Revista de la Sociedad Química de México, Zacatecas Zac., volumen 46 (número 2), noviembre 2002: 185-188.

Souza M.E. Criteria for the Utilization, Design and Operation of UASB Reactors. Water Science Technology, volumen 18 (número 12), 1986: 55-69.

Stewart. Evaluation of Septic Tank and Subsurface Flow Wetland for Jamaican Public School Wastewater Treatment, tesis (Master of Science in Environmental Engineering), Michigan, Technological University, 2005.

Van-Haandel A. y Lettinga G. Tratamento Anaeróbio de EsgotosUm Manual para Regioes de Clima Quente, EpGraf, Campina Grande-PB, Brasil, 1994.

Vázquez B., Magaña P. y Méndez N. Digestión anaerobia de aguas residuales porcícolas. Dinámica de los ácidos grasos volátiles en una planta piloto. Tecnología del agua, España, volumen XVII (número 161), 1997.

Zheng P. y Ho-Bao L. Start-up of UASB Reactor for Treatment of Pharmaceutical Wastewater. Environmental Sciences, volumen 14 (número 2), 2004: 250-254.

\section{Este artículo se cita:}

\section{Chicago citation}

Méndez-Novelo, Roger Iván, Enrique Abraham Chan-Gutiérrez, Elba René Castillo-Borges, Elizabeth del Rosario Vázquez-Borges, Arturo Edgardo Espadas-Solís. Digestión anaerobia de efluentes de fosas sépticas. Ingeniería Investigación y Tecnología, XIII, 03 (2012): 339-349.

\section{ISO 690 citation}

Méndez-Novelo R.I., Chan-Gutiérrez E.A., Castillo-Borges E.R., Vázquez-Borges E.R., Espadas-Solís A.E. Digestión anaerobia de efluentes de fosas sépticas. Ingeniería Investigación y Tecnología, volumen XIII (número 3), julio-septiembre 2012: 339-349.

\section{Semblanza de los autores}

Roger Iván Méndez-Novelo. Profesor de carrera e ingeniero civil, cuenta con especializaciones en docencia y estadística. Maestro en ingeniería ambiental y doctorado en bioquímica. Actualmente es miembro del cuerpo académico de ingeniería ambiental. Ha laborado como docente e investigador durante 30 años, ha dirigido 36 tesis de licenciatura y 16 de maestría. Ha presentado 37 ponencias y publicado 46 artículos de investigación.

Enrique Abraham Chan-Gutiérrez. Ingeniero electromecánico y maestro en ingeniería ambiental.

Elba René Castillo-Borges. Profesora de carrera e ingeniera química industrial con especialización en estadística. Maestra en ingeniería ambiental. Actualmente es coordinadora del cuerpo académico de ingeniería ambiental y del programa de maestría en ingeniería opción ambiental. Lleva 30 años como docente e investigadora, ha dirigido 8 tesis de maestría y 1 de licenciatura, ha presentado 22 ponencias y publicado 21 artículos de investigación.

Elizabeth del Rosario Vázquez-Borges. Química bióloga bromatóloga con especialización en docencia. Maestra en ingeniería ambiental y doctora en ingeniería ambiental. Tiene 25 años de experiencia docente, así como de investigación. Ha dirigido 2 tesis de licenciatura y 4 de maestría, ha presentado 12 ponencias y publicado 15 artículos de investigación. Actualmente es profesora e investigadora.

Arturo Edgardo Espadas-Solís. Ingeniero civil y miembro del cuerpo académico de hidráulica e hidrología. Cuenta con 25 años en la docencia e investigación, ha dirigido 6 tesis, ha presentado cuatro ponencias y publicado 4 artículos de investigación. Actualmente es profesor de carrera. 